\title{
Cigarette Smoke Extract and Its Cytotoxic Factor Acrolein Inhibit Nitric Oxide Production in Human Vascular Endothelial Cells
}

\author{
Takahiro Horinouchi, ${ }^{*, a}$ Yuichi Mazaki, ${ }^{a}$ Koji Terada, ${ }^{b}$ and Soichi Miwa ${ }^{a}$ \\ ${ }^{a}$ Department of Cellular Pharmacology, Graduate School of Medicine, Hokkaido University; North 15, West 7, \\ Kita-ku, Sapporo 060-8638, Japan: and ${ }^{b}$ Department of Biochemistry and Molecular Biology, Shiga University of \\ Medical Science; Seta Tsukinowa-cho, Otsu, Shiga 520-2192, Japan. \\ Received June 24, 2020; accepted August 12, 2020; advance publication released online September 1, 2020
}

Acrolein (ACR), a highly reactive $\alpha, \beta$-unsaturated aldehyde, is a major cytotoxic factor in nicotine- and tar-free cigarette smoke extract (CSE). There are conflicting results regarding endothelial functions despite the fact that both CSE and ACR cause cellular damage. Several lines of evidence indicate that CSE impairs endothelium-derived nitric oxide (NO)-dependent vasodilation by reducing the activity and protein expression of endothelial NO synthase (eNOS), whereas ACR elicits endothelium-dependent vasorelaxation by increasing the production of NO and expression of eNOS. To clarify whether CSE and its cytotoxic factor ACR cause endothelial dysfunction, this study examined the effects of CSE and ACR on human vascular endothelial EA.hy926 cells. CSE and ACR reduced the phosphorylation of eNOS at serine (Ser) ${ }^{1177}$ and total expression of eNOS. The CSE- and ACR-induced decrease in the phosphorylation and expression of eNOS was counteracted by glutathione (reduced form), an antioxidant. Basal NO production was inhibited by CSE, ACR, $N^{\mathrm{G}}$ nitro-L-arginine methyl ester (a competitive eNOS inhibitor), and nominally $\mathrm{Ca}^{2+}$-free solution supplemented with BAPTA-AM (a membrane permeable $\mathrm{Ca}^{2+}$ chelator). These results indicate that $\mathrm{CSE}$ and ACR increase oxidative stress, and reduce NO production by reducing the activity and total protein level of eNOS.

Key words acrolein; cigarette smoke extract; endothelial nitric oxide synthase; nitric oxide; endothelial dysfunction; oxidative stress

\section{INTRODUCTION}

Cigarette smoking is a major risk factor for cardiovascular diseases, such as arteriosclerosis. ${ }^{1,2}$ Cigarette mainstream smoke contains more than 4000 chemical constituents, including nicotine and particulate matter (tar). ${ }^{3)}$ Recently, we identified stable carbonyl compounds, including acrolein (ACR) and methyl vinyl ketone (MVK), as major cytotoxic factors in nicotine- and tar-free cigarette smoke extract (CSE). ${ }^{4}$ Several lines of evidence suggest that CSE, ACR, and MVK cause plasma membrane damage and cell apoptosis via $\mathrm{Ca}^{2+}$ - and protein kinase $\mathrm{C}$-dependent activation of reduced nicotinamide adenine dinucleotide phosphate (NADPH) oxidase (NOX) and the subsequent NOX-mediated generation of reactive oxygen species. ${ }^{3-6)}$

Endothelial dysfunction is characterized by (i) a reduction in endothelial nitric oxide (NO) bioavailability, which is due to the direct inactivation of NO, (ii) a decrease in the activity or expression of endothelial NO synthase (eNOS), and (iii) uncoupling of eNOS, leading to the impairment of endotheliumderived NO-dependent vasodilation. ${ }^{7-9)}$ Studies have indicated that CSE induces the impairment of endothelium-dependent vasodilation, which can be attributed to a reduction in the activity and protein expression of eNOS. ${ }^{8-10)}$ Whereas, ACR elicits endothelium-derived NO-dependent vasorelaxation by increasing eNOS expression and NO production. ${ }^{11,12)}$ These results suggest that the cigarette smoking-related endothelial dysfunction is due to cytotoxic factors other than ACR. However, the action of ACR on endothelial cells remains controversial, because ACR causes endothelial cell injury and death, both of which contribute to endothelial dysfunction. ${ }^{13,14)}$
To clarify whether CSE and ACR are involved in endothelial dysfunction, this study examined their effects on human vascular endothelial cells.

\section{MATERIALS AND METHODS}

Materials HI-LITE ${ }^{\mathrm{TM}}$ brand (Japan Tobacco Inc., Tokyo, Japan) cigarettes containing $17 \mathrm{mg}$ of tar and $1.4 \mathrm{mg}$ of nicotine per cigarette was used in the study. ACR and glutathione (reduced form, GSH) were purchased from Tokyo Chemical Industry Co., Ltd. (Tokyo, Japan). Dulbecco's modified Eagle's medium (DMEM), $N^{\mathrm{G}}$-nitro-L-arginine methyl ester (L-NAME), and 4-(2-hydroxyethyl)-1-piperazineethanesulfonic acid (HEPES) were procured from Sigma-Aldrich Co. (St. Louis, MO, U.S.A.). Fetal bovine serum (FBS) was purchased from Thermo Fisher Scientific Inc. (Waltham, MA, U.S.A.). 1,2-Bis(2-aminophenoxy)ethane- $N, N, N^{\prime}, N^{\prime}$-tetraacetic acid tetrakis(acetoxymethyl ester) (BAPTA-AM) was bought from Dojindo Laboratories (Kumamoto, Japan). 4,5-Diaminofluorescein (DAF-2) was obtained from Goryo Chemical, Inc. (Sapporo, Japan). Anti-phospho-eNOS (serine (Ser) ${ }^{1177}$ ) antibody, anti-total eNOS antibody, anti-phospho-Akt $\left(\mathrm{Ser}^{473}\right)$ antibody, and anti-total Akt antibody were procured from Cell Signaling Technology Inc. (Beverly, MA, U.S.A.). Anti-glyceraldehyde-3-phosphate dehydrogenase (GAPDH) antibody was purchased from Santa Cruz Biotechnology, Inc. (Dallas, TX, U.S.A.). Horseradish peroxidase (HRP)-conjugated anti-rabbit immunoglobulin G (IgG) secondary antibody and HRP-conjugated anti-mouse IgG secondary antibody were procured from Jackson ImmunoResearch Laboratories, Inc. (West Grove, PA, U.S.A.). The other reagents used were of the highest grade in 
A
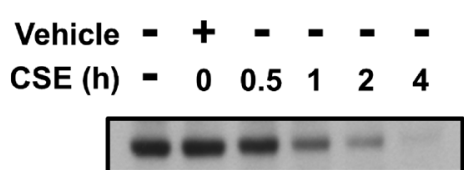

IB: $\alpha-p-e N O S$

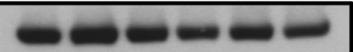

IB: $\alpha$-t-eNOS

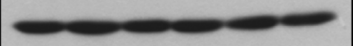

IB: $\alpha$-GAPDH

B

\section{.}

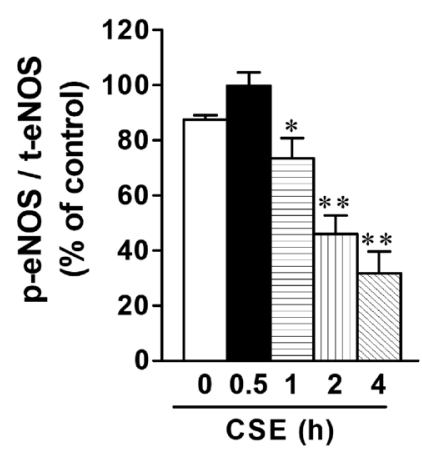

C

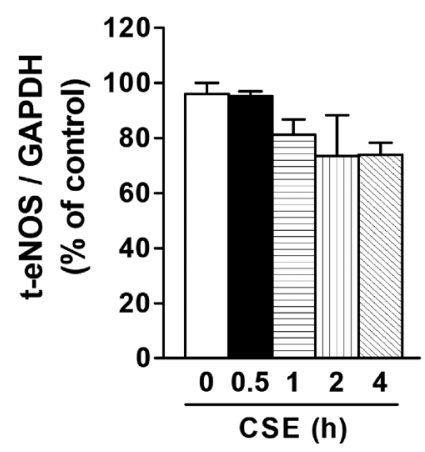

D

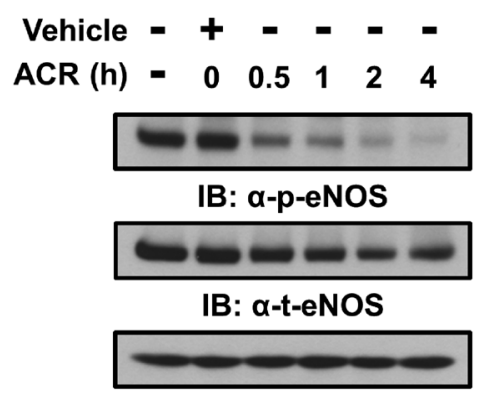

IB: $\alpha$-GAPDH

E

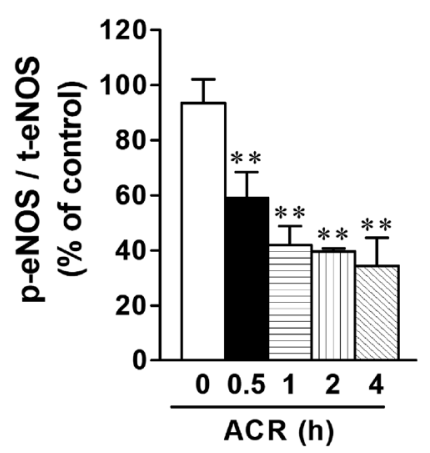

F

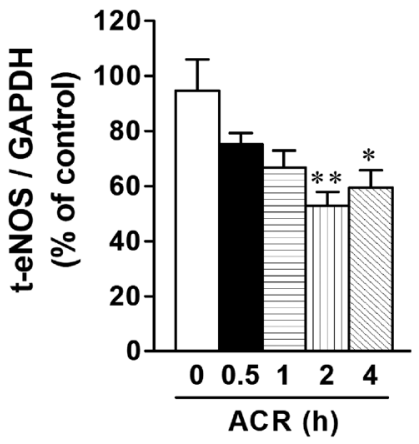

Fig. 1. Time Course of the 5\% Cigarette Smoke Extract (CSE)- and $50 \mu \mathrm{M}$ Acrolein (ACR)-Induced Decrease in the Basal Phosphorylation Level of Endothelial Nitric Oxide (NO) Synthase (eNOS) at Ser ${ }^{177}$ in Human Umbilical Vein Endothelial EA.hy926 Cells

(A, D) The upper panels are representative immunoblots with the anti-phospho-eNOS $\left(\mathrm{Ser}^{1177}\right)$ antibody to determine the level of phosphorylated eNOS (indicated as $\alpha$-p-eNOS). The middle panels are representative immunoblots with the anti-total-eNOS antibody to determine the level of total (phosphorylated and nonphosphorylated) eNOS (indicated as $\alpha$-t-eNOS). The lower panels are representative immunoblots with the anti-glyceraldehyde-3-phosphate dehydrogenase (GAPDH) antibody to determine the level of GAPDH (indicated as $\alpha-\mathrm{GAPDH}$ ) as the internal control. (B, E) The histograms present the changes in the relative ratio of the phosphorylated eNOS levels to the total eNOS expression levels (p-eNOS/t-eNOS) in the absence or presence of CSE and ACR. The phospho/total eNOS ratio was normalized by considering the ratio in control cells as $100 \%$. The data are presented as mean \pm standard error of the mean (S.E.M.) of the results obtained from four independent experiments $(* p<0.05$ and ** $p<0.01$ compared with the vehicle-treated cells). (C, F) The histograms present the changes in the relative ratio of the total eNOS levels to the total GAPDH expression levels (t-eNOS/GAPDH). The total eNOS/GAPDH ratio was normalized by considering the ratio in control cells as $100 \%$. The data are presented as mean \pm S.E.M. of the results obtained from four independent experiments $(* p<0.05$ and $* * p<0.01$ compared with the vehicle-treated cells).

terms of purity.

Preparation of CSE CSE was prepared as described previously. ${ }^{5}$ Briefly, the mainstream smoke of four cigarettes (one cigarette per trial) was continuously sucked through a standard glass-fiber Cambridge filter at a constant flow rate $\left(1.050 \mathrm{~L} \mathrm{~min}^{-1}\right)$ using an aspiration pump to remove tar and nicotine. The remaining gas phase of the cigarette smoke was bubbled into $15 \mathrm{~mL}$ of phosphate-buffered saline (PBS). The concentration of this CSE solution was considered $100 \%$.

Cell Culture Human umbilical vein endothelial EA.hy926 cells were obtained from Dr. Yuko Suzuki (Hamamatsu University School of Medicine, Japan) with permission from Dr. Cora-Jean S. Edgell (University of North Carolina at Chapel Hill, U.S.A.) and cultured in DMEM supplemented with $10 \%$ FBS (v/v), penicillin (100 units $\left.\mathrm{mL}^{-1}\right)$, and streptomycin $\left(100 \mu \mathrm{g} \mathrm{mL}^{-1}\right)$ at $37^{\circ} \mathrm{C}$ under humidified air with $5 \% \mathrm{CO}_{2}$.

Measurement of Activity and Expression of eNOS and Akt The activity and expression of eNOS and Akt were determined by Western blotting. Cells grown in six-well plates were serum-starved for $24 \mathrm{~h}$ before the experiment. The cells were treated with vehicle (PBS), CSE, or ACR in Krebs-HEPES solution $(140 \mathrm{mM} \mathrm{NaCl}, 3 \mathrm{mM} \mathrm{KCl}, 2 \mathrm{mM}$ $\mathrm{CaCl}_{2} \cdot 2 \mathrm{H}_{2} \mathrm{O}, 1 \mathrm{mM} \mathrm{MgCl} \cdot 6 \mathrm{H}_{2} \mathrm{O}, 11 \mathrm{mM} \mathrm{D}-(+)$-glucose, and $10 \mathrm{mM}$ HEPES; $\mathrm{pH}$ of the solution was adjusted to 7.4 with $\mathrm{NaOH})$. To examine the effects of $300 \mu \mathrm{M}$ GSH and its vehicle (1\% PBS) on the CSE- and ACR-induced changes in the phosphorylation and expression of eNOS and Akt, the cells were treated with GSH or vehicle for $0.5 \mathrm{~h}$ prior to adding CSE and ACR. The primary antibodies bound to proteins (phosphoeNOS $\left(\operatorname{Ser}^{1177}\right)$, total eNOS, phospho-Akt $\left(\operatorname{Ser}^{473}\right)$, total Akt, 
A
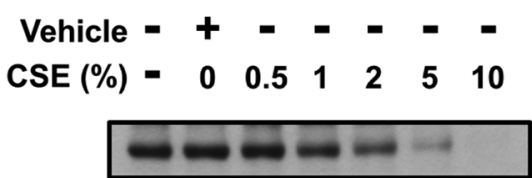

IB: a-p-eNOS

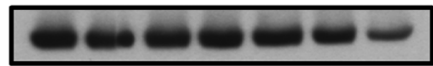

IB: a-t-eNOS

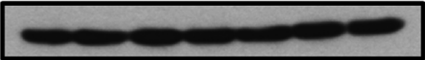

IB: $\boldsymbol{\alpha}-$ GAPDH

B

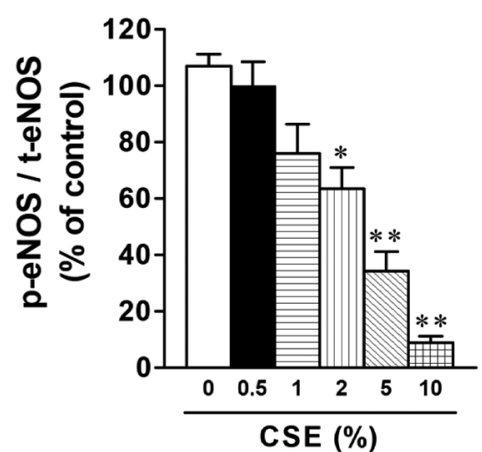

C

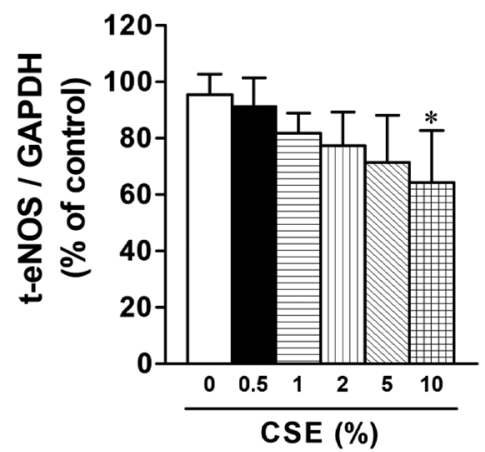

D
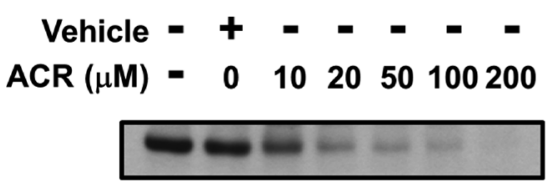

IB: $\alpha-p-e N O S$

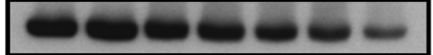

IB: $\alpha-t-e N O S$

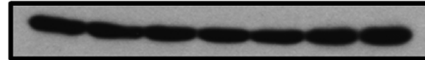

IB: $\alpha-G A P D H$

E

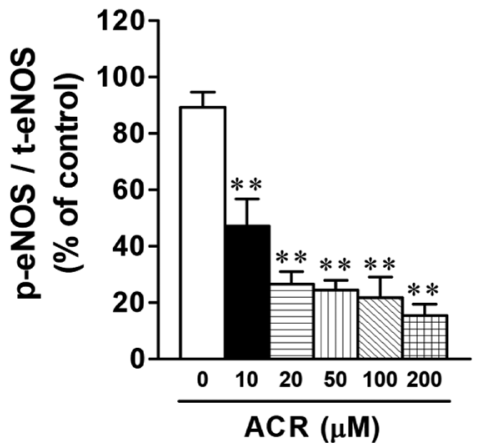

$\mathbf{F}$

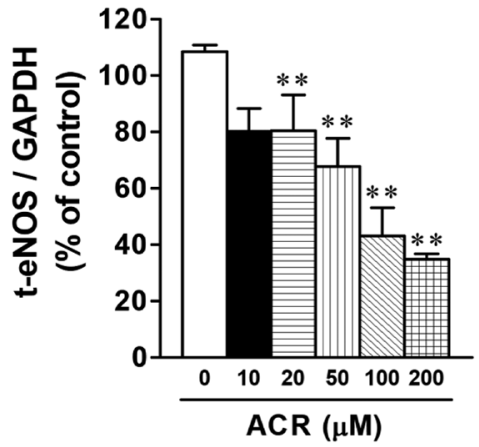

Fig. 2. Concentration-Response Relationship of the Cigarette Smoke Extract (CSE) (0.5 to 10\%)- and Acrolein (ACR) (10 to 200 $\mu$ M)-Induced Decrease in the Basal Phosphorylation Level of Endothelial Nitric Oxide Synthase (eNOS) at Ser ${ }^{1177}$ in Human Umbilical Vein Endothelial EA.hy926 Cells

(A, D) The upper panels are representative immunoblots with the anti-phospho-eNOS $\left(\mathrm{Ser}^{1777}\right)$ antibody to determine the level of phosphorylated eNOS (indicated as $\alpha$-p-eNOS). The middle panels are representative immunoblots with the anti-total-eNOS antibody to determine the level of total (phosphorylated and nonphosphorylated) eNOS (indicated as $\alpha$-t-eNOS). The lower panels are representative immunoblots with the anti-GAPDH antibody to determine the level of GAPDH (indicated as $\alpha$-GAPDH) as the internal control. (B, E) The histograms present the changes in the relative ratio of the phosphorylated eNOS levels to the total eNOS expression levels (p-eNOS/t-eNOS) in the absence or presence of CSE and ACR. The phospho/total eNOS ratio was normalized by considering the ratio in control cells as $100 \%$. The data are presented as mean \pm S.E.M. of the results obtained from four independent experiments $(* p<0.05$ and $* * p<0.01$ compared with the vehicle-treated cells). (C, F) The histograms present the changes in the relative ratio of the total eNOS levels to the total GAPDH expression levels (t-eNOS/GAPDH). The total eNOS/GAPDH ratio was normalized by considering the ratio in control cells as $100 \%$. The data are presented as mean \pm S.E.M. of the results obtained from four independent experiments $\left({ }^{*} p<0.05\right.$ and ${ }^{* *} p<0.01$ compared with the vehicle-treated cells).

and GAPDH) on the cell membrane were detected with HRPconjugated secondary antibodies and Pierce Western blotting Substrate (Thermo Fisher Scientific Inc.). The blots were exposed to Fuji medical X-ray film (FUJIFILM Corp., Tokyo, Japan). Optical density on the film was analyzed using National Institutes of Health Image J1.37 software.

Measurement of NO Production NO released from EA.hy926 cells was measured using DAF-2 (a fluorescent $\mathrm{NO}$ indicator) that reacts with NO to produce the fluorescent triazole adduct triazolofluorescein (DAF-2T). ${ }^{15)}$ Cells cultured in 24-well plates were treated with the vehicle (5\% PBS), 5\% CSE, $50 \mu \mathrm{M}$ ACR, $300 \mu \mathrm{M}$ L-NAME in Krebs-HEPES solution, or $30 \mu \mathrm{M}$ BAPTA-AM in nominally $\mathrm{Ca}^{2+}$-free Krebs-
HEPES solution for $3 \mathrm{~h}$, in the presence of $1 \mu \mathrm{M}$ DAF-2. The fluorescence intensity of the resultant DAF-2T was measured using a multi-mode spectroscopic reader (Spectra Max Paradigm; Molecular Devices, LLC., San Jose, CA, U.S.A.) at an excitation wavelength of $488 \mathrm{~nm}$ and emission wavelength of $515 \mathrm{~nm}$.

Data Analysis All results are presented as mean \pm standard error of the mean (S.E.M.) and $n$ refers to the number of experiments. All statistical analyses were performed using GraphPad Prism software (version 7.00, GraphPad Software, San Diego, CA, U.S.A.). The data were analyzed using the one-way ANOVA, followed by Tukey's multiple comparison tests. The results were considered statistically significant at a 
$p$ value of $<0.05$.

\section{RESULTS AND DISCUSSION}

Effects of CSE and ACR on eNOS Activity and Expression Previous studies on the effects of cigarette smoking on endothelium by endothelium-derived NO-mediated vasorelaxation have reported conflicting results; that is, cigarette smoking leads to augmentation or attenuation of endothelialderived NO-dependent vasorelaxation. ${ }^{16,17)}$ In vascular endothelial cells, stimulation of $\mathrm{G}_{\mathrm{q}}$ protein-coupled receptors, such as muscarinic $\mathrm{M}_{3}$ receptor and $\mathrm{Ca}^{2+}$-sensing receptor (CaSR), induces an increase in intracellular $\mathrm{Ca}^{2+}$ concentration $\left(\left[\mathrm{Ca}^{2+}\right]_{\mathrm{i}}\right)$ to activate eNOS. ${ }^{16,18)}$ eNOS is a phosphoprotein that is phosphorylated at multiple serine/threonine residues, including Ser ${ }^{1177.19)}$ The phosphorylation of eNOS at $\operatorname{Ser}^{1177}$ leads to an increase in NO production via the eNOS activity. ${ }^{20)}$ Previously, we demonstrated that the phosphorylation of eNOS at Ser ${ }^{1177}$ and production of NO are facilitated by the stimulation of CaSR with extracellular $\mathrm{Ca}^{2+}$ in human umbilical vein endothelial EA.hy926 cells. ${ }^{18)}$ To gain insights into the effect of cigarette smoke on endothelial function, here, we characterized the pharmacological properties of CSE and its cytotoxic factor ACR.

As shown in Fig. 1, the phosphorylation of eNOS at $\operatorname{Ser}^{1177}$ decreased with time after treatment with 5\% CSE (Figs. 1A, B) and $50 \mu \mathrm{M}$ ACR (Figs. 1D, E). Total eNOS protein expression was unaffected by CSE treatment (Figs. 1A, C), but it was significantly reduced by ACR treatment for 2 and $4 \mathrm{~h}$ (Figs. 1D, F). Treatment with CSE (Figs. 2A, B) and ACR (Figs. $2 \mathrm{D}, \mathrm{E})$ for $4 \mathrm{~h}$ induced a concentration-dependent decrease in the phosphorylation of eNOS. Additionally, the total eNOS protein level declined after treatment with CSE (Figs. 2A, C) and ACR (Figs. 2D, 2F). These results suggest that CSE and ACR can decrease eNOS enzyme activity by inhibiting eNOS phosphorylation, and partially, by reducing total eNOS protein expression.

Effects of CSE and ACR on NO Production To confirm whether the CSE- and ACR-induced decrease in the activity and/or expression of eNOS led to a reduction in NO generation, we measured the fluorescence intensity of DAF-2T resulting from the reaction of DAF-2 with NO. ${ }^{15}$ ) As NO is continuously generated by CaSR-mediated increases in $\left[\mathrm{Ca}^{2+}\right]_{\mathrm{i}}$ and eNOS activity in the presence of extracellular $\mathrm{Ca}^{2+18)}$ the fluorescence intensity of DAF-2T, produced due to basal NO production, was decreased by treatment with $300 \mu \mathrm{M}$ L-NAME (a competitive eNOS inhibitor) ${ }^{21)}$ and nominally $\mathrm{Ca}^{2+}$-free solution supplemented with $30 \mu \mathrm{M}$ BAPTA-AM (a membrane permeable $\mathrm{Ca}^{2+}$ chelator) ${ }^{5)}$ (Fig. 3). In addition, the fluorescent intensity of DAF-2T was significantly reduced by $5 \%$ CSE and $50 \mu \mathrm{M}$ ACR (Fig. 3). These results clearly indicate that CSE and ACR inhibit NO production. Our findings support the concept that cigarette smoking is deleterious to endothelial function. However, our results regarding the action of ACR contradict those of a previous study in which ACR stimulated NO production via an increase in $\left[\mathrm{Ca}^{2+}\right]_{\mathrm{i}}$ in human umbilical vein endothelial cells. ${ }^{11)}$ The reason for this discrepancy in the results is unknown, but it may be due to the difference in the type of endothelial cells used (primary cultured cells in the previous study ${ }^{11}$ versus immortalized EA.hy926 cells in this study). Alternatively, CaSR-mediated responses observed in

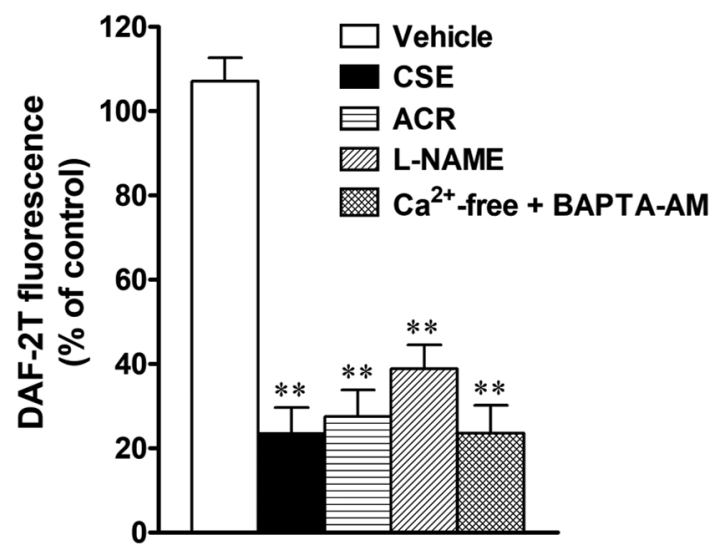

Fig. 3. Effects of $2 \%$ PBS (vehicle), $5 \%$ CSE, $50 \mu \mathrm{M} \mathrm{ACR,} 300 \mu \mathrm{M} \mathrm{L}$ NAME, and Nominally $\mathrm{Ca}^{2+}$-Free Solution Supplemented with $30 \mu \mathrm{M}$ BAPTA-AM on NO Production in Human Umbilical Vein Endothelial EA.hy926 Cells

NO released from EA.hy926 cells was measured using DAF-2, which reacts with NO to produce its fluorescent triazole adduct DAF-2T. The fluorescence intensity of DAF-2T was calculated by subtracting the fluorescence intensity obtained in the absence of cells from that obtained in the presence of cells. The data are presented as mean \pm S.E.M. of results obtained from seven independent experiments $\left(*^{*} p<0.01\right.$ compared with the vehicle-treated cells).

EA.hy926 cells may have masked ACR-induced NO production if ACR has opposing effects on endothelial function-for example, stimulation and inhibition of NO generation.

Effects of GSH on the CSE- and ACR-Induced Changes in Phosphorylation and Expression of eNOS and Akt CSE and ACR can induce oxidative stress that contributes to endothelial dysfunction. ${ }^{13)}$ It has been reported that antioxidants such as GSH and $N$-acetyl-L-cysteine reduce the cytotoxicity of CSE and ACR. ${ }^{5,13)}$ Indeed, $300 \mu \mathrm{M}$ GSH inhibited the $5 \%$ CSE- and $50 \mu \mathrm{M}$ ACR-induced decreases in the phosphorylation and expression of eNOS (Figs. 4A-C). These results suggest that oxidative stress caused by CSE and ACR leads to a decline in the eNOS phosphorylation and expression.

Does ACR play a central role in the CSE-induced endothelial dysfunction? Several studies have indicated that ACR is one of the substances present in high level in CSE. ${ }^{3,4,13)}$ We have reported that the concentration of ACR in the CSE, which was prepared by dissolving the gas phase of 10 cigarettes in $10 \mathrm{~mL}$ of water, was approximately $3.4 \mathrm{mM}^{4)}$ It means that the estimated amount of ACR generated is $3.4 \mu \mathrm{mol}$ per one cigarette. Therefore, the concentration of ACR in 5\% CSE prepared in this study (see Materials and Methods) is estimated as $45 \mu \mathrm{M}$. As shown in Figs. 3 and 4B, 5\% CSE was as effective as $50 \mu \mathrm{M}$ ACR in terms of the reduction of eNOS phosphorylation and NO production. These results, taken together with the estimated concentration of ACR in 5\% CSE, indicate that the inhibitory effect of CSE on the eNOS/NO axis is mainly due to ACR.

However, the phosphorylation of $\mathrm{Ser}^{473}$ of Akt, which is an upstream kinase of eNOS and phosphorylates Ser $^{1177}$ of eNOS, ${ }^{22)}$ were slightly reduced by $5 \%$ CSE but significantly elevated by $50 \mu \mathrm{M}$ ACR (Figs. 4D, E). Interestingly, 5\% CSE enhanced the Akt phosphorylation in the presence of $300 \mu \mathrm{M}$ GSH (Figs. 4D, E). Fifty micromolar ACR induced a decrease in Akt expression, which was recovered by $300 \mu \mathrm{M}$ GSH (Figs. 4D, F). These results indicate that ACR per se activates Akt, while oxidizing substance(s) other than ACR present in the CSE inhibits Akt activation. Further studies will be 
A

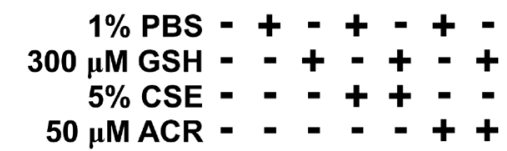

IB: a-p-eNOS

IB: $\alpha-t-e N O S$

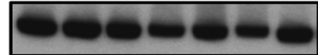

IB: $\alpha-G A P D H$

B

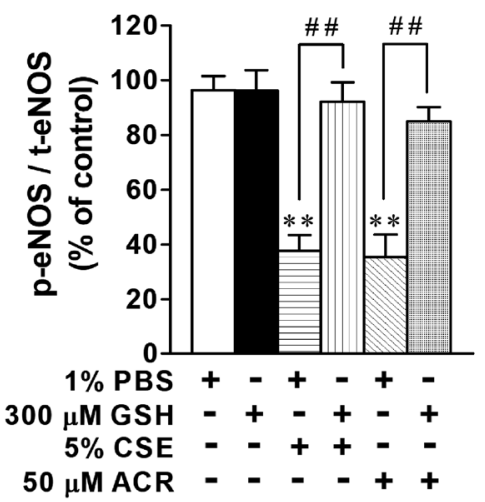

C

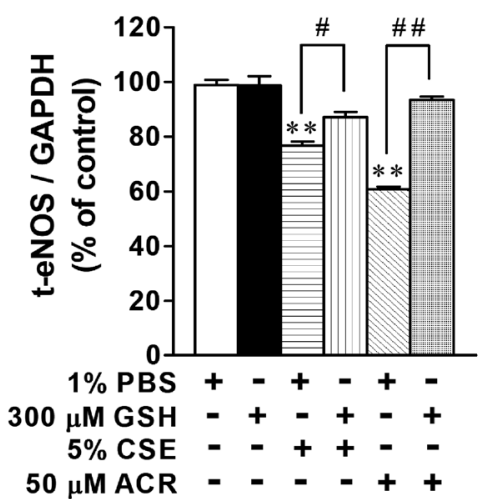

D

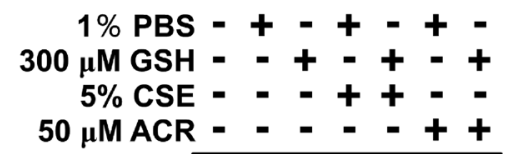

IB: $\alpha-p-A K t$

IB: $\alpha-t-A k t$

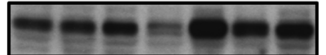

IB: $\alpha-G A P D H$

E

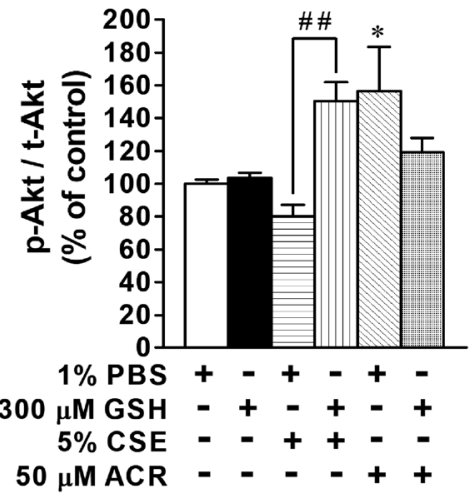

F

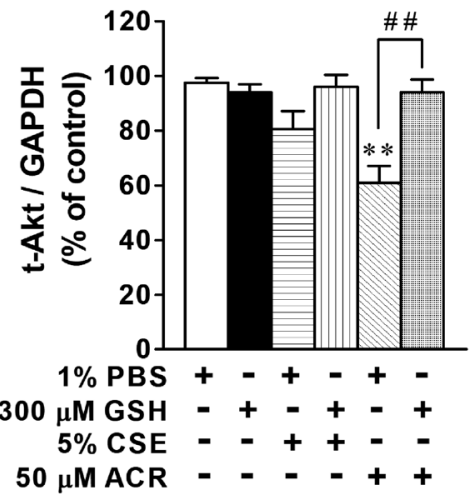

Fig. 4. Effects of $1 \%$ PBS (Vehicle) and $300 \mu \mathrm{M}$ Glutathione (Reduced Form, GSH) on the $5 \%$ CSE- and $50 \mu \mathrm{M}$ ACR-Induced Changes in the Basal Phosphorylation Levels of Endothelial Nitric Oxide Synthase (eNOS) at $\operatorname{Ser}^{1177}$ (A-C) and Akt at Ser ${ }^{473}$ (D-F) in Human Umbilical Vein Endothelial EA.hy926 Cells

(A, D) The upper panels are representative immunoblots with the anti-phospho-eNOS $\left(\mathrm{Ser}^{1177}\right)$ antibody and the anti-phospho-Akt (Ser $\left.{ }^{473}\right)$ antibody to determine the levels of phosphorylated eNOS (indicated as $\alpha$-p-eNOS) and phosphorylated Akt (indicated as $\alpha$-p-Akt), respectively. The middle panels are representative immunoblots with the anti-total-eNOS antibody and the anti-total-Akt antibody to determine the levels of total (phosphorylated and nonphosphorylated) eNOS (indicated as $\alpha$-t-eNOS) and total Akt (indicated as $\alpha$-t-Akt), respectively. The lower panels are representative immunoblots with the anti-GAPDH antibody to determine the level of GAPDH (indicated as $\alpha$-GAPDH) as the internal control. (B, E) The histograms present the changes in the relative ratio of the phosphorylated eNOS or Akt levels to the total eNOS or Akt expression levels (p-eNOS/t-eNOS or p-Akt/t-Akt) in the absence or presence of CSE and ACR. The phospho/total eNOS or Akt ratio was normalized by considering the ratio in control cells as $100 \%$. The data are presented as mean \pm S.E.M. of the results obtained from four independent experiments $(* p<0.05$ and $* * p<0.01$ compared with the vehicle-treated cells; ${ }^{\#} p<0.01$, between indicated columns). (C, F) The histograms present the changes in the relative ratio of the total eNOS or Akt levels to the total GAPDH expression levels (t-eNOS/GAPDH or t-Akt/GAPDH). The total eNOS or Akt/GAPDH ratio was normalized by considering the ratio in control cells as $100 \%$. The data are presented as mean \pm S.E.M. of the results obtained from four independent experiments $\left(* * p<0.01\right.$ compared with the vehicle-treated cells; ${ }^{*} p<0.05$ and ${ }^{\# \#} p<0.01$, between indicated columns).

required to determine how $\mathrm{CSE}$ and $\mathrm{ACR}$ modulate the Akt/ eNOS/NO pathway.

\section{CONCLUSION}

The present study provides evidence that CSE and ACR increase oxidative stress, and decrease NO production by reducing the activity and/or total protein level of eNOS. These findings suggest that (i) cigarette smoking leads to the attenuation of endothelial-derived NO-dependent vasorelaxation, and (ii) antioxidant compounds such as GSH may be effective in inhibiting cigarette smoking-related endothelial dysfunction.
Acknowledgments We thank Dr. Cora-Jean S. Edgell (University of North Carolina at Chapel Hill, U.S.A.) and Dr. Yuko Suzuki (Hamamatsu University School of Medicine, Japan) for supplying EA.hy926 cells. This study was partially supported by Grants-in-Aid for Scientific Research (C) from Japan Society for the Promotion of Science [No. 18K06697 (to T. H.)] and Smoking Research Foundation of Japan (to T. H. and S. M.).

Conflict of Interest The authors declare no conflict of interest. 


\section{REFERENCES}

1) IARC. Tabacco smoke and involuntary smoking. IARC Monogr. Eval. Carcinog. Risks Hum., 83, 1-1438 (2004).

2) Smith CJ, Fischer TH. Particulate and vapor phase constituents of cigarette mainstream smoke and risk of myocardial infarction. Atherosclerosis, 158, 257-267 (2001).

3) Horinouchi T, Higashi T, Mazaki Y, Miwa S. Carbonyl compounds in the gas phase of cigarette mainstream smoke and their pharmacological properties. Biol. Pharm. Bull., 39, 909-914 (2016).

4) Noya Y, Seki K, Asano H, Mai Y, Horinouchi T, Higashi T, Terada K, Hatate C, Hoshi A, Nepal P, Horiguchi M, Kuge Y, Miwa S. Identification of stable cytotoxic factors in the gas phase extract of cigarette smoke and pharmacological characterization of their cytotoxicity. Toxicology, 314, 1-10 (2013).

5) Asano H, Horinouchi $T$, Mai Y, Sawada O, Fujii S, Nishiya T, Minami M, Katayama T, Iwanaga T, Terada K, Miwa S. Nicotineand tar-free cigarette smoke induces cell damage through reactive oxygen species newly generated by PKC-dependent activation of NADPH oxidase. J. Pharmacol. Sci., 118, 275-287 (2012).

6) Mai Y, Higashi $T$, Terada K, Hatate C, Nepal P, Horiguchi M, Harada T, Miwa S, Horinouchi T. Nicotine- and tar-free cigarette smoke extract induces cell injury via intracellular $\mathrm{Ca}^{2+}$-dependent subtype-specific protein kinase C activation. J. Pharmacol. Sci., 120, 310-314 (2012).

7) Landmesser U, Hornig B, Drexler H. Endothelial function: a critical determinant in atherosclerosis? Circulation, 109 (Suppl. 1), II-27II-33 (2004).

8) $\mathrm{Su}$ Y, Han W, Giraldo C, De Li Y, Block ER. Effect of cigarette smoke extract on nitric oxide synthase in pulmonary artery endothelial cells. Am. J. Respir. Cell Mol. Biol., 19, 819-825 (1998).

9) Raveendran M, Wang J, Senthil D, Wang J, Utama B, Shen Y, Dudley D, Zhang Y, Wang XL. Endogenous nitric oxide activation protects against cigarette smoking induced apoptosis in endothelial cells. FEBS Lett., 579, 733-740 (2005).

10) Abdelghany TM, Ismail RS, Mansoor FA, Zweier JR, Lowe F, Zweier JL. Cigarette smoke constituents cause endothelial nitric oxide synthase dysfunction and uncoupling due to depletion of tetrahydrobiopterin with degradation of GTP cyclohydrolase. Nitric Oxide, 76, 113-121 (2018).

11) Misonou Y, Asahi M, Yokoe S, Miyoshi E, Taniguchi N. Acrolein produces nitric oxide through the elevation of intracellular calcium levels to induce apoptosis in human umbilical vein endothelial cells: implications for smoke angiopathy. Nitric Oxide, 14, 180-187 (2006).

12) Awe SO, Adeagbo AS, D'Souza SE, Bhatnagar A, Conklin DJ. Acrolein induces vasodilatation of rodent mesenteric bed via an EDHF-dependent mechanism. Toxicol. Appl. Pharmacol., 217, 266-276 (2006)

13) Zirak MR, Mehri S, Karimani A, Zeinali M, Hayes AW, Karimi G. Mechanisms behind the atherothrombotic effects of acrolein, a review. Food Chem. Toxicol., 129, 38-53 (2019).

14) Madamanchi NR, Vendrov A, Runge MS. Oxidative stress and vascular disease. Arterioscler. Thromb. Vasc. Biol., 25, 29-38 (2005).

15) Ghafourifar P, Parihar MS, Nazarewicz R, Zenebe WJ, Parihar A Detection assays for determination of mitochondrial nitric oxide synthase activity; advantages and limitations. Methods Enzymol., 440, 317-334 (2008).

16) Nene S, Gelabert H, Moore W, Quinones-Baldrich W, SantibanezGallerani A, Ignarro L. Cigarette smoking increases endothelialderived vasorelaxation in the rat carotid artery in a dose-dependent manner. J. Surg. Res., 71, 101-106 (1997).

17) Toda N, Okamura T. Cigarette smoking impairs nitric oxidemediated cerebral blood flow increase: implications for Alzheimer's disease. J. Pharmacol. Sci., 131, 223-232 (2016).

18) Horinouchi T, Mazaki Y, Terada K, Miwa S. Extracellular $\mathrm{Ca}^{2+}$ promotes nitric oxide production via $\mathrm{Ca}^{2+}$-sensing receptor- $\mathrm{G}_{\mathrm{a} / 1}$ protein-endothelial nitric oxide synthase signaling in human vascular endothelial cells. J. Pharmacol. Sci., 143, 315-319 (2020).

19) Dudzinski DM, Igarashi J, Greif D, Michel T. The regulation and pharmacology of endothelial nitric oxide synthase. Annu. Rev. Pharmacol. Toxicol., 46, 235-276 (2006).

20) McCabe TJ, Fulton D, Roman LJ, Sessa WC. Enhanced electron flux and reduced calmodulin dissociation may explain "calciumindependent" eNOS activation by phosphorylation. J. Biol. Chem., 275, 6123-6128 (2000).

21) Kopincová J, Púzserová A, Bernátová I. L-NAME in the cardiovascular system-nitric oxide synthase activator? Pharmacol. Rep., 64, 511-520 (2012).

22) Ning WH, Zhao K. Propionyl-L-carnitine induces eNOS activation and nitric oxide synthesis in endothelial cells via PI3 and Akt kinases. Vascul. Pharmacol., 59, 76-82 (2013). 